\title{
Inhibiting NTRK2 signaling causes endometriotic lesion regression
}

\author{
Hsiu-Chi Lee®1', Shih-Chieh Lin², Meng-Hsing Wu² and Shaw-Jenq Tsai® ${ }^{2,3}$ \\ ${ }^{1}$ Department of Obstetrics and Gynecology, College of Medicine, National Cheng Kung University, Tainan, Taiwan, \\ ${ }^{2}$ Institute of Basic Medical Sciences, College of Medicine, National Cheng Kung University, Tainan, Taiwan and \\ ${ }^{3}$ Department of Physiology, College of Medicine, National Cheng Kung University, Tainan, Taiwan
}

Correspondence should be addressed to S-J Tsai; Email: seantsai@mail.ncku.edu.tw

\begin{abstract}
Endometriosis is a common gynecological disease in reproductive-age women. Although the hormone-dependent therapy is the first line treatment for endometriosis, it is not a curative regimen and associated with severe side-effects, which significantly decrease the life quality of affected individuals. To seek a target for treatment of endometriosis, we focused on plasma membrane proteins that are elevated in ectopic cells and exert beneficial effects in cell growth and survival. We performed bioinformatics analysis and identified the neurotrophic receptor tyrosine kinase 2 (NTRK2) as a potential candidate for treatment. The expression levels of NTRK2 were markedly upregulated in the lesions of clinical specimen as well as in the mouse endometriotic-like lesion. Mechanistic investigation demonstrated that upregulation of NTRK2 is induced by hypoxia in a hypoxia-inducible factor 1 alpha-dependent manner.

Knockdown of NTRK2 or administration of ANA-12, a selective antagonist of NTRK2, significantly induced endometriotic stromal cells death, suggesting it may be a potential therapeutic agent. In vivo study using surgery-induced endometriosis mice model showed ANA-12 (1.5 mg/kg body weight) treatment induced apoptosis of endometriotic cells and caused the regression of ectopic lesions. Taken together, our findings suggest a possible mechanism responsible for the aberrant expression of NTRK2 in endometriotic lesions and this may be involved in the pathogenesis of endometriosis.
\end{abstract}

Reproduction (2021) 161 11-19

\section{Introduction}

Endometriosis is a common gynecological disease defined as the growth of endometrial tissue outside of the uterus. About ten percent of women are persecuted by endometriosis. The clinical symptoms of this disease are chronic pelvic pain, dyspareunia, dysmenorrhea, and infertility; however, the exact cause of endometriosis is complex and largely unclear. Presently, the hormonebased treatments and surgical intervention are two main options for relieving the symptoms (Ferrero et al. 2018). Nevertheless, there are significant unmet needs due to the unfavorable side effects accompanied with the hormonal medication and the high recurrence after the surgery.

The etiology of endometriosis is incompletely understood, nevertheless, the retrograde menstruation theory has been proposed since 1927. In the menstrual period, endometrial cells flow back through the fallopian tubes and into the peritoneal cavity (Sampson 1927). To date, numerous evidences indicate that hypoxic peritoneal cavity and various growth factors, including cytokines and estrogen in the peritoneal fluid, can facilitate ectopic cell growth in the peritoneal cavity (Oosterlynck et al. 1994, Pizzo et al. 2002, Bulun 2009,
Wu et al. 2010, Young et al. 2013, Hsiao et al. 2015). Thus, characterizing mediators in the peritoneal fluid and peritoneal tissues may provide valuable information to identify potential candidate genes contributing to the pathophysiological processes of endometriosis. However, the components of peritoneal fluid are too complex to dissect mechanisms leading to the development of endometriosis. Therefore, an alternative way is to identify gene expression change between the eutopic and ectopic cells to find critical genes involved in promoting endometriosis development.

Neurotrophic receptor tyrosine kinase 2 (NTRK2), also known as tropomyosin receptor kinase B (TrkB), is a membrane-bound receptor for brain-derived neurotrophic factor (BDNF) and neurotrophin-4 (NTF4). Upon the ligand binding, NTRK2 homodimerizes, autophosphorylates itself, and activates the downstream cascades. NTRK2 not only involves in the neuron differentiation through the GRB2-Ras-MAPK cascade but also regulates growth and survival through multiple signaling pathway (Yang et al. 2019). NTRK2 is expressed in many tissues, especially in brain tissues. It has been reported that heterozygous mutations of NTRK2 can cause neurodevelopmental disorder such as early infantile epileptic encephalopathy-58 (EIEE58) 
(Hamdan et al. 2017), and a disease named obesity, hyperphagia, and developmental delay (Yeo et al. 2004, Miller et al. 2017). The fusion of NTRK2 with $Q K I$ due to chromosome translocation, $\mathrm{t}(6: 9)$, results in the formation of pilocytic astrocytoma (Jones et al. 2013). Genetically engineered mouse study showed that mutations on different sites of Ntrk2 possess unique and conserved biological functions in taste system (Koudelka et al. 2014). Moreover, the variants (Luberg et al. 2010) and SNP (Velez Edwards et al. 2013, Metrustry et al. 2014) also contribute to the complexity of the molecular functions and pathological impacts NTRK2 exerts.

The expression and function of BDNF/NTRK2 in reproductive system and related diseases were less investigated. BDNF mRNA was found to be increased in ovarian endometrioma (Borghese et al. 2010) but the protein level was not different in peritoneal fluid of women with endometriosis (Barcena de Arellano et al. 2013). In addition, it was reported that the serum mature BDNF is not a useful biomarker for endometriosis (Perricos et al. 2018). Previously, increased NTRK2 expression was observed in the deep infiltrating endometriosis (Dewanto et al. 2016). Recent study indicates that NTRK2 is increased in the secretory endometrium of women with adenomyosis and positively correlates with the serum CA125 concentration and dysmenorrhea (Huang et al. 2011). However, the function of NTRK2 in endometriosis remains unclear.

In this study, we demonstrated that NTRK2 expression was elevated in the ectopic endometriotic tissues and endometriotic stromal cells. In addition, we revealed the functional characteristics of NTRK2 in the endometriosis. Furthermore, we showed that inhibition of NTRK2 could be the alternative for treatment of endometriosis.

\section{Materials and methods}

\section{Clinical specimens}

Endometrial and endometriosis tissues were obtained from the Department of Obstetrics and Gynecology of National Cheng Kung University Hospital under a procedure approved by the Clinical Research Ethics Committee at the National Cheng Kung University Medical Center. Endometriosis was confirmed via the clinical diagnosis and pathology examination by a gynecologist and a pathologist. The revised American Society for Reproductive Medicine scoring system has been followed to classify the stages of endometriosis. Fifty-nine endometriosis specimens (paired tissues $=26$, non-paired endometriotic tissues $=33$ ) were obtained from women with mild endometriosis (stage II), moderate (stage III) or severe (stage IV) endometriosis (Supplementary Table 1, see section on supplementary materials given at the end of this article). Written informed consent was obtained from each patient.

\section{Cell culture and treatments}

Experiments were performed in the isolated stromal cells from the endometrial and endometriotic tissues. The procedure for isolation of stromal cells was as previously described (Lin et al. 2019). In brief, tissues were digested with type IV collagenase $(2 \mathrm{mg} / \mathrm{mL})$ and DNase I $(100 \mu \mathrm{g} / \mathrm{mL})$ in PBS and shaken with $250 \mathrm{~g}$ for $60 \mathrm{~min}$ at $37^{\circ} \mathrm{C}$. The stromal cells were selected through $70 \mu \mathrm{m}$ pore size and then $40 \mu \mathrm{m}$ pore size nylon cell strainer. Filtered cells were allowed to attach for $30 \mathrm{~min}$ in a T-75 flask. After $30 \mathrm{~min}$, the tissue debris, blood cells and epithelial cells were removed by washing with PBS. For the purity, we routinely stained the isolated endometrial stromal cells with vimentin (positive marker) and keratin (epithelial cell marker for negative control) antibodies. Cells were cultured in DMEM/nutrient mixture F-12 (DMEM/F12) supplemented with $10 \%$ fetal bovine serum (FBS), 1× GlutaMAX (Life Technologies, Inc) and $1 \times$ Antibiotic-Antimycotic Solution (Caisson Laboratories, Inc, North Logan, Utah) in a humidified atmosphere with $5 \% \mathrm{CO}_{2}$ at $37^{\circ} \mathrm{C}$.

In the cytokines and estradiol $\left(E_{2}\right)$ stimulation experiment, eutopic stromal cells were cultured in the phenol red-free DMEM/F12 medium supplemented with charcoal-stripped 1\% FBS and various stimuli for $24 \mathrm{~h}$. In the hypoxia treatment, eutopic stromal cells were cultured with DMEM/F12 supplemented with $10 \%$ FBS in a hypoxia chamber $\left(1 \% \mathrm{O}_{2}\right.$, $94 \% \mathrm{~N}_{2}$ ) for $24 \mathrm{~h}$.

\section{Bioinformatics analysis}

The expression profiles of endometriosis were downloaded from gene expression omnibus (GEO). We used the GSE23339 (Hawkins et al. 2011) and the GSE25628 (Crispi et al. 2013) datasets. The data were first analyzed using GEO2R for comparing the normal and endometriosis groups. After analysis, results were saved in a txt file format. The txt file was opened by excel and then selected the upregulating genes $(P<0.05$, fold change $\geq 1.5$ ). On the other hand, human genes with receptor activity were downloaded from AmiGO2 (MunozTorres \& Carbon 2017) by using gene ontology term 'signaling receptor activity' (ID: GO:0038023, Alt ID: GO:0004872) with the following filters: organism: Homo sapiens, contributor: Uniprot. A total of 1289 genes were retrieved. Upregulated genes from two different endometriosis datasets and gene list of receptor were uploaded to a website tool (the Venn diagrams tool; http://bioinformatics.psb.ugent.be/webtools/Venn/) and the intersection of three datasets (GSE23339, GSE25628 and receptor gene list) were generated. The $P$ values of the interest genes were calculated by an $\mathrm{R}$ package, SuperExactTest (https://cran.r-project.org/web/packages/SuperExactTest/index. html) (Wang et al. 2015). To evaluate the expression of NTRK2, another dataset GSE7305 (Hever et al. 2007) that contains mRNA expression levels of normal and diseased endometrial tissues were also downloaded for analysis.

\section{Small interfering RNA (siRNA) knockdown}

Eutopic stromal cells were transfected with either control siRNA or siRNA targeted to HIF- $1 \alpha$ using Lipofetamine 2000 
according to the manufacturer's instructions. After $24 \mathrm{~h}$ incubation, the cells were incubated under either normoxia or hypoxia $\left(1 \% \mathrm{O}_{2}\right)$ for another $24 \mathrm{~h}$. Cells were then harvested for evaluation of mRNA expression level.

Ectopic stromal cells were transfected with either control siRNA or NTRK2 siRNA using Lipofetamine 2000, and then plated at a density of $2 \times 10^{5}$ cells per well in the six-well plate for further cell apoptosis assay.

\section{$R N A$ isolation and reverse transcriptase quantitative polymerase chain reaction ( $R T-q P C R)$}

Total RNA was prepared from the cells according to the manufacturer's instructions (TRIsure; Bioline, London, United Kingdom). Five hundred nanogram of total RNA was reverse transcribed into cDNA and then quantified using realtime PCR to determine the gene expression levels. FastStart Universal SYBR Green Master (Roche) and the specific primers were used to amplify genes of interest. The $18 \mathrm{~S}$ rRNA was used for normalization. The sequences of the primers used were: NTRK2: forward 5'-GGGACACCACGAACAGAAGT-3'; reverse 5'-CACCACAGCATAGACCGAGA-3'; BDNF: forward 5'-ATTCAGCCCATTTCACACCT-3'; reverse 5'-TTTGCTTATCCСТCACCCTAC-3'; $18 \mathrm{~S}$ rRNA: forward 5'- GTGTGCCTACCCTACG-3'; reverse 5'TGACCCGCACTTACTG-3'.

\section{Immunocytochemistry}

Sections of paraffin-embedded endometria were processed according to standard protocol. The sections were deparaffinized and rehydrated with xylene and serial ethanol $(100,95,80$, and $70 \%)$ followed by washing in deionized water. Sections underwent antigen retrieval in citric buffer (10 $\mathrm{mM}, \mathrm{pH}$ 6.0) and immerged in Protein Block from Novolink Polymer Detection Systems (Leica Biosystems) for $1 \mathrm{~h}$ at room temperature to block nonspecific binding. Primary antibody against NTRK2 $(1: 250$, Santa Cruz) or phosphorylated NTRK2 (pTyr515) were incubated with sections in humidified chamber at $4{ }^{\circ} \mathrm{C}$ overnight. If the host of primary antibody is mouse, then the post primary block was added for $1 \mathrm{~h}$ at room temperature, followed by incubation with Novocasta polymer for $1 \mathrm{~h}$ at room temperature. If the host of primary antibody is rabbit, the sections were directly incubated with Novocasta polymer for $1 \mathrm{~h}$ at room temperature. Sections were washed and then developed by PolyDetector Liquid AEC HRP (Bio SB, Santa Barbara, California). The slide was counterstained with hematoxylin. Tissue sections were mounted on coverslips using glycerol gelatin mounting medium (Sigma).

For evaluating the staining, Histoscore (H-score) was calculated by a semi-quantitative assessment of staining intensities in positively-stained stromal cells $(3 \times$ percentage of strongly staining $+2 \times$ percentage of moderately staining + percentage of weakly staining nuclei, giving a range of 0 to 300) performed by two individuals. Or, the values were calculated by ImageJ, which assessed the staining area and intensity. It was showed as (positive staining area $\times$ intensity)/ total area.

\section{Apoptosis assay}

Ectopic cells were treated with siRNA or different doses of ANA12 (Sigma). After the treatment, both the floating and adhesive cells were collected and stained with annexin-V (BioLegend, San Diego, California) for $20 \mathrm{~min}$ at room temperature in dark. The annexin- $V$ positive cells were determined using FACSCanto II Flow Cytometer (BD, Franklin Lakes, New Jersey) and analyzed using Flowjo ${ }^{\circledR}$ software (Flowjo, Ashland, Oregon).

\section{Mouse model of endometriosis}

Female C57BL/6NCrj mice (8-10 weeks old) were purchased from the Animal Center at the College of Medicine, National Cheng Kung University. To establish the mouse model of endometriosis, an estrogen capsule was imbedded in the female mouse back for 2 days. After $E_{2}$ stimulation, one side of the uterus was removed and divided into four equal-size pieces. Then the fragmented uterus was inoculated on the peritoneal wall in the same mouse for 1 month. Mice were then grouped randomly to receive vehicle or $1.5 \mathrm{mg} / \mathrm{kg}$ body weight ANA-12 compound intraperitoneally three times a week for additional 3 weeks. The weights of ectopic lesions in both groups were measured.

\section{TUNEL assay}

In vivo apoptosis detection was performed in the endometriotic-like lesions according to the manufacturer's instructions (Abcam plc). Sections of paraffin-embedded lesions were processed according to standard protocol. The sections were deparaffinized and rehydrated with xylene and serial ethanol $(100,95,80$, and $70 \%)$ followed by washing in $1 \times$ PBS. Permeabilization of specimen was performed by using proteinase K. Following by inactivation of endogenous peroxidases and equilibration, and then $\mathrm{TdT}$ labeling reaction was performed at room temperature for $1.5 \mathrm{~h}$. After incubation, the entire specimen was covered by stop buffer and incubated at room temperature for $5 \mathrm{~min}$ followed by incubating with blocking buffer for $10 \mathrm{~min}$. After blocking, $1 \times$ conjugate was added to the specimen and incubated for $30 \mathrm{~min}$. DAB solution was used for color development and hematoxylin was used for counterstain. Tissue sections were mounted on coverslips using glycerol gelatin mounting medium (Sigma).

\section{Statistical analysis}

Results are expressed as mean \pm S.E.M.and were analyzed using GraphPad Prism version 6 (GraphPad Software). Student's $t$-test was used to compare two groups or one-way ANOVA was used to compare three or more groups. The post-test analysis was performed using Dunnett's multiple comparisons test. A $P$ value less than 0.05 was considered statistically significant.

\section{Results}

\section{NTRK2 is increased in the endometriotic lesions}

Toscreen a targetcandidatefor treatment of endometriosis, we analyzed the gene expression omnibus (GEO) 
datasets and cell membrane receptors list in view of the important role of cellular stimulus responses. We crossreferenced the upregulated genes in two GEO (GSE23339 and GSE25628) datasets and cell membrane receptors list (Fig. 1A). Nineteen candidates overexpressed in the endometriotic specimens were identified. NTRK2 is one of the candidates involved in the process of cell survival and proliferation. In addition, we further verified the expression of NTRK2 in another endometriosis GEO dataset (GSE7305). The results of the three GEO datasets (GSE23339, GSE25628, and GSE7305) indicated that NTRK2 was increased in the endometriotic lesions (Fig. 1B). Therefore, we hypothesized that NTRK2 may play a role in the development of endometriosis.

To determine whether NTRK2 signaling is involved in endometriosis pathogenesis, we quantified the expression levels of NTRK2 and its ligand, BDNF in eutopic and ectopic endometriotic cells. The results demonstrated that expression of NTRK2 and BDNF was elevated in the ectopic lesions (Fig. 1C) and primary culture endometriotic stromal cells (Fig. 1D) as compared to the eutopic counterparts. We further conducted the immunohistochemical staining for NTRK2 in clinical specimen. The results demonstrate that expression of NTRK2 was significantly increased in the ectopic lesions (Fig. 1E) even when paired samples were compared (Fig. 1F). Furthermore, the elevated level of NTRK2 was independent of menstrual cycle (Fig. 1G). These results indicated that BDNF-NTRK2 signaling cascade may play a role in the development of endometriosis.

\section{Elevated expression of NTRK2 in the ectopic stromal cell was mediated by hypoxia}

We then investigated the underlying mechanisms responsible for NTRK2 overexpression in endometriotic cells. As endometriosis is an inflammatory and estrogendependent disease, we treated endometrial stromal cells with proinflammatory cytokines (including IL- $1 \beta$, TNF $\alpha$, TGF $\beta$, and IL-6) and estradiol $\left(\mathrm{E}_{2}\right)$. However, these stimuli did not increase the expression level of NTRK2 (Fig. 2A). We then examined whether aberrant expression of NTRK2 is mediated by hypoxia since it has been implicated to play critical roles in endometriosis (Hsiao et al. 2015, Wu et al. 2019). The result showed that the expression level of NTRK2 was elevated under hypoxic condition (Fig. 2B). In addition, knockdown of hypoxia inducible factor $1 \alpha$ (HIF- $1 \alpha$ ) attenuated hypoxiainduced NTRK2 expression (Fig. 2C), indicating hypoxia induces NTRK2 is mediated at the transcriptional level.

\section{Inhibition of NTRK2 signaling induces apoptosis of the ectopic stromal cells}

To understand the effect of the increased NTRK2 expression in the ectopic stromal cells, NTRK2 was knocked down by siRNA and cells were cultured for
48 h. The data showed that knockdown of NTRK2 increased number of apoptotic cells (Fig. 3A). Next, we treated ectopic endometriotic stromal cells with serial doses of ANA-12, a selective inhibitor for NTRK2 but not NTRK1 or NTRK3 (Cazorla et al. 2011), and found ANA-12 significantly induced apoptosis at the dose of $25 \mu \mathrm{M}$ (Fig. 3B).

\section{Administration of NTRK2 causes endometriotic lesion regression in mouse model of endometriosis}

To examine the potential of ANA-12 for treatment of endometriosis, mouse model of endometriosis was employed (Fig. 4A). Level of NTRK2 is dramatically upregulated in ectopic lesions of mice (Fig. 4A). Treatment with ANA-12 (1.5 mg/kg body weight) reduced the volume of endometriosis-like lesions as compared to vehicle-treated group (Fig. 4B). We determined the phosphorylated NTRK2 (Tyr515), the activated NTRK2, in the ectopic lesions and found the ANA-12 treatment tended to decrease the phosphorylation of NTRK2 (Fig. 4C). The in situ apoptotic analysis showed that treatment with ANA-12 induced apoptosis in endometriotic-like lesion but not in the eutopic endometrium (Fig. 4D).

\section{Discussion}

In the present study, we found NTRK2 was upregulated in ectopic endometriotic tissues and stromal cells compared to its eutopic counterpart likely due to hypoxic stress. Treatment with NTRK2 antagonist, ANA12 , induced apoptosis in endometriotic stromal cells and caused the regression of the endometriosis-like lesion in model animals. These data suggest aberrant expression of NTRK2 exerts protective effect of endometriotic stromal cells and warrant further investigation to explore the possibility of using NTRK2 as a molecular target for treating endometriosis.

NTRK2 is a conserved gene in human, chimpanzee, Rhesus monkey, dog, cow, mouse and is known as the BDNF receptor. It has been reported that the level of NTRK2 is higher in eutopic endometrium in women with endometriosis (Anger et al. 2007), adenomyosis (Huang et al. 2011), and deep infiltrating endometriosis (Matsuzaki et al. 2004), but not in ovarian endometriosis (Matsuzaki et al. 2006). Our results showed that NTRK2 was increased in ovarian endometriosis (Fig. 1 and Supplementary Table 1). Furthermore, our finding demonstrated that hypoxia induces NTRK2 through transcriptional regulation by HIF- $1 \alpha$. The reason that our result is different from that of Matsuzaki's (Matsuzaki et al. 2006) may be due to different methods were used for evaluation. Matsuzaki et al. used RT-qPCR to detect the mRNA transcripts of NTRK2 while we performed IHC to identified NTRK2 protein. As there are various alternative splicing variants of NTRK2 (Luberg et al. 2010, Fenner 2012), the concordance of transcripts and 
A

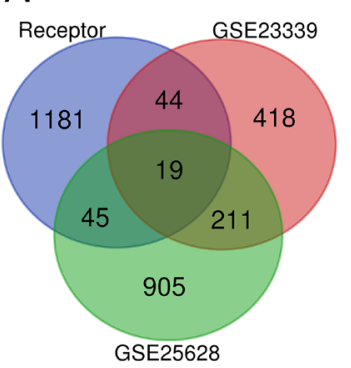

C



E

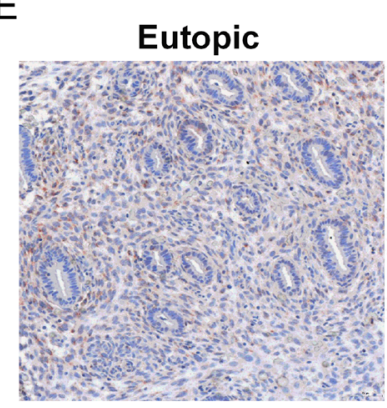

F

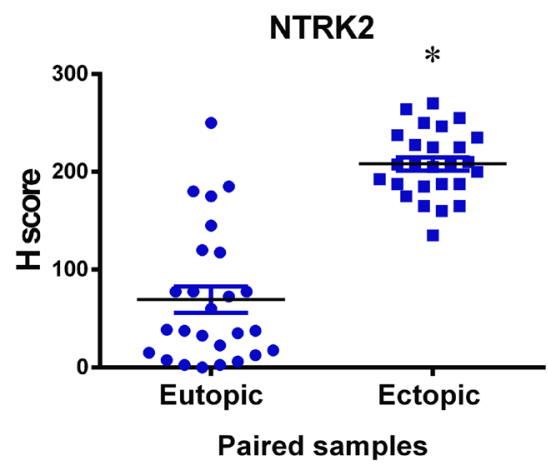

B
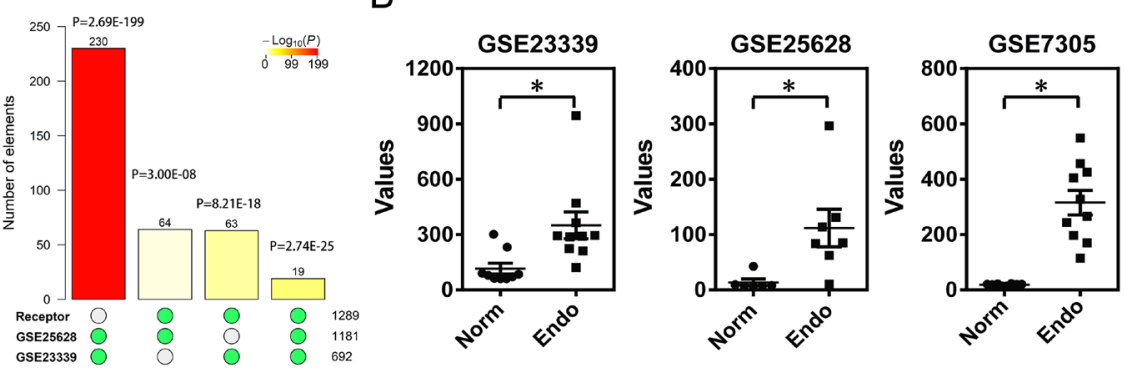

D
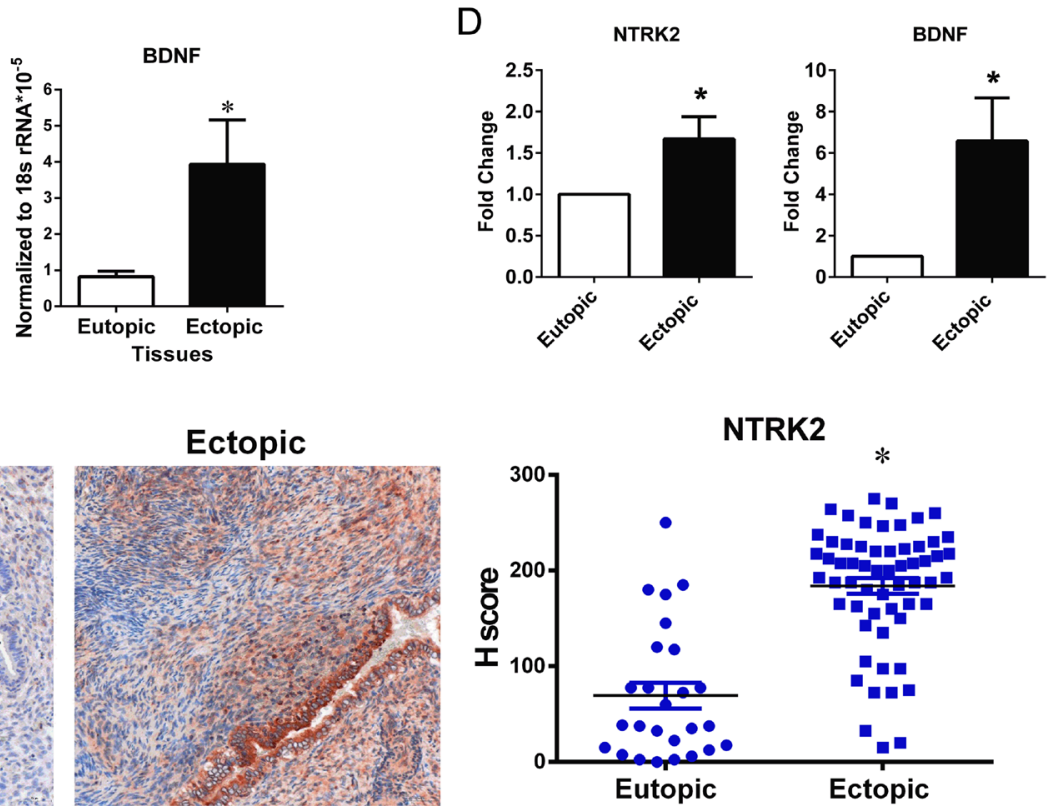

G

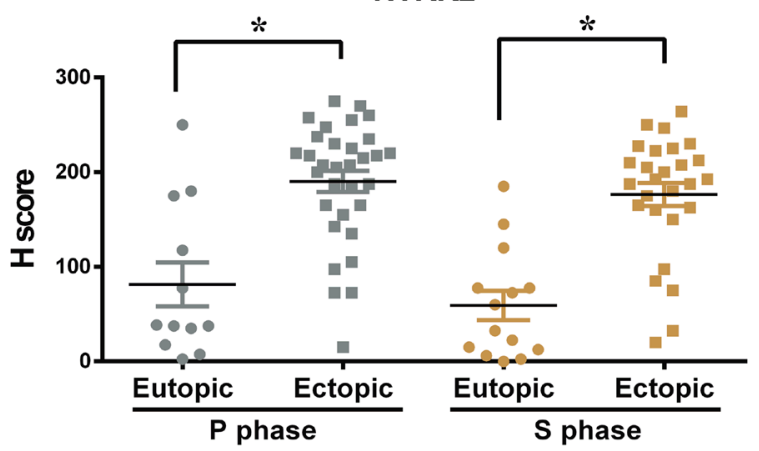

Figure 1 NTRK2 expression level is elevated in ectopic tissues. (A) Genes upregulated in endometriosis $(P<0.05$ and fold change $>1.5)$ derived from public datasets (GSE23339 and GSE25628) were cross-referenced with a membrane receptor gene list from GO annotation. Venn diagram (left) and landscape chart with $P$ values (right) were analyzed by Bioinformatic tools as described in materials and methods. (B) NTRK2 levels were analyzed in three public datasets (GSE23339, GSE 25628, and GSE7395). (C) The mRNA levels of NTRK2 were detected in paired endometrial tissues $(n=10)$ and (D) paired endometrial stromal cells from patients with endometriosis $(n=7)$. The levels of NTRK2 and BDNF mRNA were normalized to $18 \mathrm{~S}$ rRNA. (E) NTRK2 expression levels were detected in clinical specimen $(n=26$ for eutopic tissues, $n=59$ for ectopic tissues) by immunohistochemistry. The same concentration of IgM antibody was hybridized as negative control. Quantification of the IHC result was shown in right panel. (F) Quantification of IHC result for paired tissues $(n=26)$. (G) Analysis of the NTRK2 expression level in the different phases of menstrual cycle. Asterisks indicated significant difference at $P<0.05$; Norm, normal endometrium; Endo, endometriosis;

P phase, proliferative phase; $\mathrm{S}$ phase, secretory phase. 

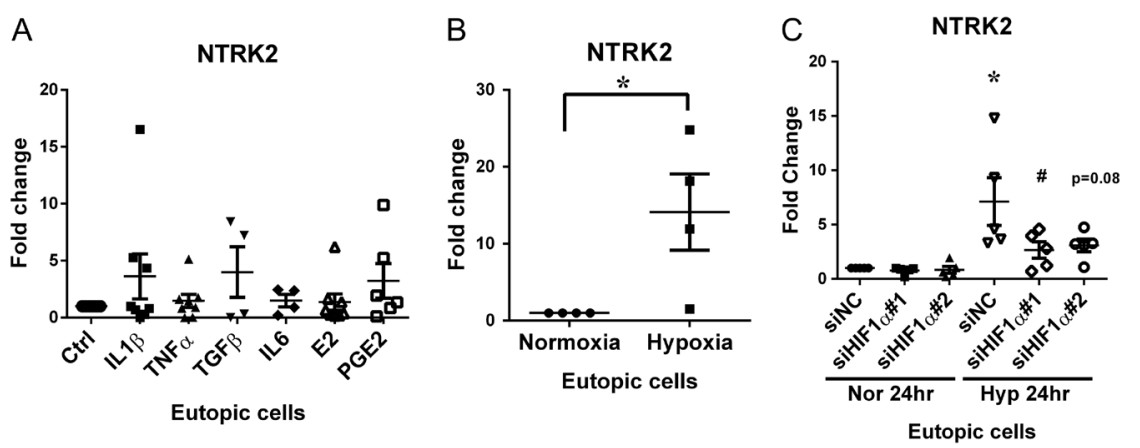

Figure 2 The NTRK2 induction is mediated by hypoxia. (A) The mRNA level of NTRK2 was detected in various cytokines and $\mathrm{E}_{2}$-treated eutopic endometrial stromal cells $(n=8)$. (B) NTRK2 level was measured in normoxia or hypoxia-treated eutopic stromal cells $(n=4)$. (C) Eutopic stromal cells were transfected with control siRNA or siRNA against HIF- $1 \alpha$ and incubated under either normoxia or hypoxia for $24 \mathrm{~h}$ ( $n=5$ biological repeats). The levels of NTRK2 mRNA were normalized to $18 \mathrm{~S}$ rRNA. ${ }^{*} P<0.05 ;{ }^{*} P<0.05$ compared to hypoxia control siRNA group. IL1 $\beta(1 \mathrm{ng} / \mathrm{mL})$, interleukin-1 beta; TNF $\alpha(10 \mathrm{ng} / \mathrm{mL})$, tumor necrosis factor-alpha; TGF $\beta(10 \mathrm{ng} / \mathrm{mL})$, transforming growth factor beta 1; IL6 (10 ng/mL), interleukin-6; $E_{2}(1 \mathrm{nM})$, estradiol; $\mathrm{PGE}_{2}(1 \mathrm{nM})$, Prostaglandin $\mathrm{E}_{2}$; Nor, normoxia; Hyp, hypoxia. proteins is ambiguous. Therefore, it is better and more representative to directly measure the level of protein instead of mRNA.

Although previous studies showed distinct expression of NTRK2 between normal endometrial and endometriotic cells (Matsuzaki et al. 2004, 2006, Anger et al. 2007, Huang et al. 2011); however, what causes NTRK2 overexpression in endometriotic cells has never been reported. Herein, we tested several potential factors that had been shown to play critical roles in the pathogenesis of endometriosis such as estradiol, $\mathrm{PGE}_{2}$, and proinflammatory cytokines $\mathrm{Wu}$ et al. 2010, Hsiao et al. 2015, Li et al. 2018) and found they did not contribute to aberrant expression of NTRK2 in endometriotic stromal cells. Our finding is consistent with a previous report that estradiol failed to affect NTRK2 expression (Wessels et al. 2015). On the other hand, we demonstrated that hypoxia is the critical factor inducing the expression of NTRK2. Since hypoxia is suggested to be the driving force of endometriosis (Wu et al. 2019), our current finding reveals another pathological process regulated by hypoxia.

BDNF-NTRK2 signaling cascades control neuron differentiation, growth, survival, and synaptic plasticity (Kaplan \& Miller 2000, Huang \& Reichardt 2001, 2003, Minichiello 2009). In endometriosis, effect of BDNF-
NTRK2 was reported to be associated with endometriosisrelated pain (Barcena de Arellano et al. 2013, Ding et al. 2018). Our data showed that BDNF-NTRK2 signaling is important for anti-apoptosis as knockdown of NTRK2 increased apoptosis of endometriotic stromal cells. This finding agrees with that observed in cancer cells as accumulated evidence indicates that NTRK2 promotes tumor cell survival, drug resistance, and metastasis (Yu et al. 2008, Yuan et al. 2018). Based on this finding, we reasoned that blocking BDNF-NTRK2 signaling may be an alternative way to treat endometriosis. Indeed, treatment with ANA-12 enhanced the apoptosis of ectopic stromal cells. Furthermore, ANA-12 administration leads to endometriotic lesion regression in autotransplanted mouse model. Result from TUNEL assay indicates blocking NTRK2 signaling by ANA-12 increased apoptotic cell number in ectopic endometriotic lesion. Interestingly, intact eutopic endometrial cells were not affected by ANA-12 treatment in the same mouse. The reason for differential responses to ANA-12 is unknown and warrants further investigation.

ANA-12 was identified as a selective NTRK2 antagonist, which has been reported to reduce chronic pain in different experimental models (Tillu et al. 2015, Liu et al. 2018, Fan et al. 2020). Furthermore, ANA-12 has been used to target NTRK2 in studies
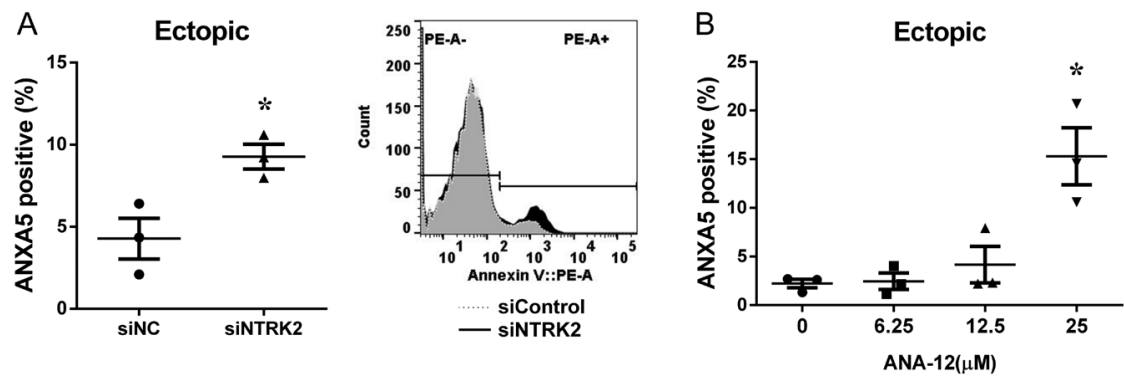

Figure 3 Elevated NTRK2 expression possesses the ability to promote anti-apoptosis of ectopic stromal cell. (A) The flow cytometry was performed in the ectopic stromal cells treated with control siRNA or NTRK2 siRNA for $48 \mathrm{~h}$ $(n=3)$. (B) Ectopic endometriotic stromal cells were treated with ANA-12 for $48 \mathrm{~h}$. The cells were collected and stained with annexin- $\mathrm{V}$ as an apoptotic marker and analyzed using flow cytometry $(n=3) . * P<0.05$ indicates significant different compared to control group. ANXA5 indicates Annexin A5 (or annexin $\mathrm{V}$ ). 

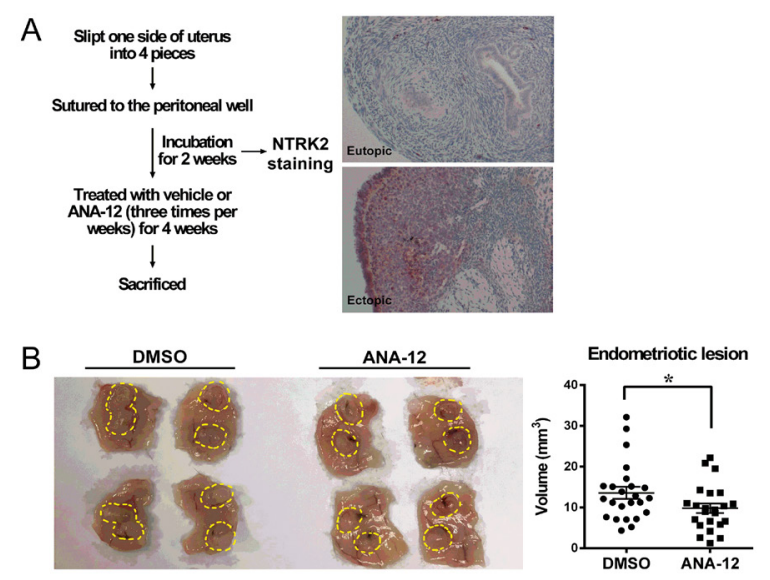

C


Figure 4 Specific NTRK2 inhibitor decreases the lesion growth in the endometriosis mouse model. (A) The diagram showing the procedures used to set up the animal model of endometriosis (left). The NTRK2 level was determined in surgical-induced mouse model using IHC staining (right). (B) Left panel: representative picture of ectopic lesions after DMSO or ANA-12 treatment $(1.5 \mathrm{mg} / \mathrm{kg}$ body weight) for 1 month. The yellow dot-line indicated the location of lesions. Right panel: the volume of ectopic lesion was measured when mice were sacrificed (DMSO, $n=24$; ANA-12, $n=23$ ). (C) The level of phosphorylated NTRK2 (Tyr515) was detected in the ectopic lesions after DMSO or ANA-12 treatment (DMSO, $n=24$; ANA-12, $n=23$ ). (D) Apoptotic levels were determined in the ectopic lesions and uteri after DMSO or ANA-12 treatment (DMSO: lesion, $n=24$, uterus, $n=6$; ANA-12: $n=23$, uterus, $n=6)$. Asterisks indicate $P<0.05$.

of medulloblastoma (Thomaz et al. 2019), gliomas (Pinheiro et al. 2017), leukemia (Polakowski et al. 2014), lung adenocarcinoma metastasis (Sinkevicius et al. 2014) and lymphoid tissue neovascularization (Dalton et al. 2015). In our study, we demonstrated that ANA-12 treatment decreased the lesion size and increased the cell death in lesion but not in eutopic endometrium, suggesting that targeting BDNF-NTRK2 pathway may be a possible alternative for treating endometriosis. However, there are some limitations of current study. First, the ANA-12 was administered to an artificial mouse model of endometriosis, in which uterine tissue was sutured in the peritoneal cavity. This model may not recapitulate the real tissue microenvironment at the time of retrograde menstruation and true inflammatory responses. In addition, despite that ANA-12 treatment induced about two -folds increase in cell death, the reduction of lesion size is not as great, suggesting other factors may be involved in lesion regression. Thus, the impact on the peritoneal microenvironment of ANA-12 administration needs further exploration.

In conclusion, NTRK2 is aberrantly expressed in endometriotic stromal cells due to hypoxic stress and contributes to anti-apoptosis. Treatment with selective NTRK2 antagonist causes regression of endometrioticlike lesion in mouse model suggests targeting NTRK2 signaling might be a potential choice for non-hormonal treatment of endometriosis.

\section{Supplementary materials}

This is linked to the online version of the paper at https://doi. org/10.1530/REP-20-0163.

\section{Declaration of interest}

The authors declare that there is no conflict of interest that could be perceived as prejudicing the impartiality of the research reported.

\section{Funding}

This work was supported by grants from Ministry of Science and Technology of Taiwan (MOST 104-2320-B-006-036-MY3 and MOST 106-2320-B-006-072-MY3).

\section{Author contribution statement}

H C Lee and S C Lin conducted the experiment, collected and analyzed data. $\mathrm{M} \mathrm{HWu}$ and S J Tsai conceived the project and provided guidance of clinical and basic medical sciences, respectively. H C Lee and S J Tsai wrote the manuscript.

\section{Acknowledgements}

We wish to express our appreciation to Miss Mei-Feng Huang, Miss Yi-Hsuan Yeh, and Miss Yi-Chen Tang for assistance with primary endometrial cell isolation, IHC staining and scoring, and animal experiments. We also thank Center of Bioinformatics and Digital Health for providing professional help in Bioinformatic analysis.

\section{References}

Anger DL, Zhang B, Boutross-Tadross O \& Foster WG 2007 Tyrosine receptor kinase $\mathrm{B}(\mathrm{TrkB})$ protein expression in the human endometrium. Endocrine 31 167-173. (https://doi.org/10.1007/s12020-007-0025-8)

Barcena de Arellano ML, Arnold J, Lang H, Vercellino GF, Chiantera V, Schneider A \& Mechsner S 2013 Evidence of neurotrophic events due to peritoneal endometriotic lesions. Cytokine 62 253-261. (https://doi. org/10.1016/j.cyto.2013.03.003) 
Borghese B, Vaiman D, Mondon F, Mbaye M, Anaf V, Noel JC, de Ziegler D \& Chapron C 2010 Neurotrophins and pain in endometriosis. Gynecologie, Obstetrique and Fertilite 38 442-446. (https://doi. org/10.1016/j.gyobfe.2010.05.005)

Bulun SE 2009 Endometriosis. New England Journal of Medicine $\mathbf{3 6 0}$ 268-279. (https://doi.org/10.1056/NEJMra0804690)

Cazorla M, Premont J, Mann A, Girard N, Kellendonk C \& Rognan D 2011 Identification of a low-molecular weight TrkB antagonist with anxiolytic and antidepressant activity in mice. Journal of Clinical Investigation 121 1846-1857. (https://doi.org/10.1172/JCl43992)

Crispi S, Piccolo MT, D'Avino A, Donizetti A, Viceconte R, Spyrou M, Calogero RA, Baldi A \& Signorile PG 2013 Transcriptional profiling of endometriosis tissues identifies genes related to organogenesis defects. Journal of Cellular Physiology 228 1927-1934. (https://doi.org/10.1002/ jcp.24358)

Dalton JE, Glover AC, Hoodless L, Lim EK, Beattie L, Kirby A \& Kaye PM 2015 The neurotrophic receptor Ntrk2 directs lymphoid tissue neovascularization during Leishmania donovani infection. PLoS Pathogens 11 e1004681. (https://doi.org/10.1371/journal. ppat.1004681)

Dewanto A, Dudas J, Glueckert R, Mechsner S, Schrott-Fischer A, Wildt L \& Seeber B 2016 Localization of TrkB and p75 receptors in peritoneal and deep infiltrating endometriosis: an immunohistochemical study. Reproductive Biology and Endocrinology 14 43. (https://doi.org/10.1186/ s12958-016-0178-5)

Ding S, Zhu T, Tian Y, Xu P, Chen Z, Huang X \& Zhang X 2018 Role of brain-derived neurotrophic factor in endometriosis pain. Reproductive Sciences 25 1045-1057. (https://doi.org/10.1177/1933719117732161)

Fan F, Tang Y, Dai H, Cao Y, Sun P, Chen Y, Chen A \& Lin C 2020 Blockade of BDNF signalling attenuates chronic visceral hypersensitivity in an IBS-like rat model. European Journal of Pain 24 839-850. (https://doi. org/10.1002/ejp.1534)

Fenner BM 2012 Truncated TrkB: beyond a dominant negative receptor. Cytokine and Growth Factor Reviews 23 15-24. (https://doi. org/10.1016/j.cytogfr.2012.01.002)

Ferrero S, Barra F \& Roberti Maggiore UL 2018 Current and Emerging Therapeutics for the management of endometriosis. Drugs 78 995-1012. (https://doi.org/10.1007/s40265-018-0928-0)

Hamdan FF, Myers CT, Cossette $\mathrm{P}$, Lemay $\mathrm{P}$, Spiegelman $\mathrm{D}$, Laporte $\mathrm{AD}$, Nassif C, Diallo O, Monlong J, Cadieux-Dion M et al. 2017 High rate of recurrent de novo mutations in developmental and epileptic encephalopathies. American Journal of Human Genetics 101 664-685. (https://doi.org/10.1016/j.ajhg.2017.09.008)

Hawkins SM, Creighton CJ, Han DY, Zariff A, Anderson ML, Gunaratne PH \& Matzuk MM 2011 Functional microRNA involved in endometriosis. Molecular Endocrinology 25 821-832. (https://doi.org/10.1210/ me.2010-0371)

Hever A, Roth RB, Hevezi P, Marin ME, Acosta JA, Acosta H, Rojas J, Herrera R, Grigoriadis D, White E et al. 2007 Human endometriosis is associated with plasma cells and overexpression of B lymphocyte stimulator. PNAS 104 12451-12456. (https://doi.org/10.1073/ pnas.0703451104)

Hsiao KY, Lin SC, Wu MH \& Tsai SJ 2015 Pathological functions of hypoxia in endometriosis. Frontiers in Bioscience 7 309-321. (https://doi. org/10.2741/e736)

Huang EJ \& Reichardt LF 2001 Neurotrophins: roles in neuronal development and function. Annual Review of Neuroscience $\mathbf{2 4}$ 677-736. (https://doi.org/10.1146/annurev.neuro.24.1.677)

Huang EJ \& Reichardt LF 2003 Trk receptors: roles in neuronal signal transduction. Annual Review of Biochemistry 72 609-642. (https://doi. org/10.1146/annurev.biochem.72.121801.161629)

Huang Y, Zheng W, Mu L, Ren Y, Chen X \& Liu F 2011 Expression of tyrosine kinase receptor $\mathrm{B}$ in eutopic endometrium of women with adenomyosis. Archives of Gynecology and Obstetrics 283 775-780. (https://doi.org/10.1007/s00404-010-1718-4)

Jones DT, Hutter B, Jager N, Korshunov A, Kool M, Warnatz HJ, Zichner T, Lambert SR, Ryzhova M, Quang DA et al. 2013 Recurrent somatic alterations of FGFR1 and NTRK2 in pilocytic astrocytoma. Nature Genetics 45 927-932. (https://doi.org/10.1038/ng.2682)

Kaplan DR \& Miller FD 2000 Neurotrophin signal transduction in the nervous system. Current Opinion in Neurobiology 10 381-391. (https:// doi.org/10.1016/s0959-4388(00)00092-1)
Koudelka J, Horn JM, Vatanashevanopakorn C \& Minichiello L 2014 Genetic dissection of TrkB activated signalling pathways required for specific aspects of the taste system. Neural Development 9 21. (https:// doi.org/10.1186/1749-8104-9-21)

Li WN, Wu MH \& Tsai SJ 2018 Critical factors involved in chronic inflammation in the pathophysiology of endometriosis. Adaptive Medicine 10 1-9. (https://doi.org/10.4247/AM.2018.ABI197)

Lin SC, Lee HC, Hsu CT, Huang YH, Li WN, Hsu PL, Wu MH \& Tsai SJ 2019 Targeting anthrax toxin receptor 2 ameliorates endometriosis progression. Theranostics 9 620-632. (https://doi.org/10.7150/thno.30655)

Liu C, Zhang Y, Liu Q, Jiang L, Li M, Wang S, Long T, He W, Kong X, Qin G et al. 2018 P2X4-receptor participates in EAAT3 regulation via BDNF-TrkB signaling in a model of trigeminal allodynia. Molecular Pain 14 1744806918795930. (https://doi.org/10.1177/1744806918795930)

Luberg K, Wong J, Weickert CS \& Timmusk T 2010 Human TrkB gene: novel alternative transcripts, protein isoforms and expression pattern in the prefrontal cerebral cortex during postnatal development. Journal of Neurochemistry 113 952-964. (https://doi.org/10.1111/j.14714159.2010.06662.x)

Matsuzaki S, Canis M, Vaurs-Barriere C, Pouly JL, Boespflug-Tanguy O, Penault-Llorca F, Dechelotte P, Dastugue B, Okamura K \& Mage G 2004 DNA microarray analysis of gene expression profiles in deep endometriosis using laser capture microdissection. Molecular Human Reproduction 10 719-728. (https://doi.org/10.1093/molehr/gah097)

Matsuzaki S, Canis M, Pouly JL, Botchorishvili R, Dechelotte PJ \& Mage G 2006 Differential expression of genes in eutopic and ectopic endometrium from patients with ovarian endometriosis. Fertility and Sterility 86 548-553. (https://doi.org/10.1016/j.fertnstert.2006.02.093)

Metrustry SJ, Edwards MH, Medland SE, Holloway JW, Montgomery GW, Martin NG, Spector TD, Cooper C \& Valdes AM 2014 Variants close to NTRK2 gene are associated with birth weight in female twins. Twin Research and Human Genetics 17 254-261. (https://doi.org/10.1017/thg.2014.34)

Miller KA, Twigg SR, McGowan SJ, Phipps JM, Fenwick AL, Johnson D, Wall SA, Noons P, Rees KE, Tidey EA et al. 2017 Diagnostic value of exome and whole genome sequencing in craniosynostosis. Journal of Medical Genetics 54 260-268. (https://doi.org/10.1136/ jmedgenet-2016-104215)

Minichiello L 2009 TrkB signalling pathways in LTP and learning. Nature Reviews: Neuroscience 10 850-860. (https://doi.org/10.1038/ nrn2738)

Munoz-Torres M \& Carbon S 2017 Get go! Retrieving GO data using AmiGO, QuickGO, API, files, and tools. Methods in Molecular Biology 1446 149-160. (https://doi.org/10.1007/978-1-4939-3743-1 11)

Oosterlynck DJ, Meuleman C, Waer M \& Koninckx PR 1994 Transforming growth factor-beta activity is increased in peritoneal fluid from women with endometriosis. Obstetrics and Gynecology 83 287-292.

Perricos A, Ashjaei K, Husslein H, Proestling K, Kuessel L, Obwegeser R, Wenzl R \& Yotova I 2018 Increased serum levels of mBDNF in women with minimal and mild endometriosis have no predictive power for the disease. Experimental Biology and Medicine 243 50-56. (https://doi. org/10.1177/1535370217742600)

Pinheiro KV, Alves C, Buendia M, Gil MS, Thomaz A, Schwartsmann G, de Farias CB, Roesler R, Bowman RL, Wang Q et al. 2017 Targeting tyrosine receptor kinase B in gliomas. Neuro-Oncology 19 138-139. (https://doi. org/10.1093/neuonc/now199)

Pizzo A, Salmeri FM, Ardita FV, Sofo V, Tripepi M \& Marsico S 2002 Behaviour of cytokine levels in serum and peritoneal fluid of women with endometriosis. Gynecologic and Obstetric Investigation 54 82-87. (https://doi.org/10.1159/000067717)

Polakowski N, Terol M, Hoang K, Nash I, Laverdure S, Gazon H, Belrose G, Mesnard JM, Cesaire R, Peloponese JM et al. 2014 HBZ stimulates brain-derived neurotrophic factor/TrkB autocrine/paracrine signaling to promote survival of human T-cell leukemia virus type 1-Infected T cells. Journal of Virology 88 13482-13494. (https://doi.org/10.1128/ JVI.02285-14)

Sampson JA 1927 Metastatic or embolic endometriosis, due to the menstrual dissemination of endometrial tissue into the venous circulation. American Journal of Pathology 3 93-110.43 143.

Sinkevicius KW, Kriegel C, Bellaria KJ, Lee J, Lau AN, Leeman KT, Zhou P, Beede AM, Fillmore CM, Caswell D et al. 2014 Neurotrophin receptor TrkB promotes lung adenocarcinoma metastasis. PNAS 111 10299-10304. (https://doi.org/10.1073/pnas.1404399111) 
Thomaz A, Pinheiro KV, Souza BK, Gregianin L, Brunetto AL, Brunetto AT, de Farias CB, Jaeger MDC, Ramaswamy V, Nor C et al. 2019 Antitumor activities and cellular changes induced by TrkB inhibition in medulloblastoma. Frontiers in Pharmacology 10 698. (https://doi. org/10.3389/fphar.2019.00698)

Tillu DV, Hassler SN, Burgos-Vega CC, Quinn TL, Sorge RE, Dussor G, Boitano S, Vagner J \& Price TJ 2015 Protease-activated receptor 2 activation is sufficient to induce the transition to a chronic pain state. Pain 156 859-867. (https://doi.org/10.1097/j.pain.0000000000000125)

Velez Edwards DR, Naj AC, Monda K, North KE, Neuhouser M, Magvanjav O, Kusimo I, Vitolins MZ, Manson JE, O'Sullivan MJ et al. 2013 Gene-environment interactions and obesity traits among postmenopausal African-American and Hispanic women in the Women's Health Initiative SHARe Study. Human Genetics 132 323-336. (https:// doi.org/10.1007/s00439-012-1246-3)

Wang M, Zhao Y \& Zhang B 2015 Efficient test and visualization of multiset intersections. Scientific Reports 5 16923. (https://doi.org/10.1038/ srep16923)

Wessels JM, Leyland NA, Agarwal SK \& Foster WG 2015 Estrogen induced changes in uterine brain-derived neurotrophic factor and its receptors. Human Reproduction 30 925-936. (https:/doi.org/10.1093/humrep/ dev018)

Wu MH, Lu CW, Chuang PC \& Tsai SJ 2010 Prostaglandin E2: the master of endometriosis? Experimental Biology and Medicine 235 668-677. (https://doi.org/10.1258/ebm.2010.009321)

Wu MH, Hsiao KY \& Tsai SJ 2019 Hypoxia: the force of endometriosis. Journal of Obstetrics and Gynaecology Research 45 532-541. (https:// doi.org/10.1111/jog.13900)
Yang SP, Lo CY, Tseng HM \& Chao CC 2019 Knockdown of protein kinase CK2 blocked gene expression mediated by brain-derived neurotrophic factor-induced serum response element. Chinese Journal of Physiology 62 63-69. (https://doi.org/10.4103/CJP.CJP_1_19)

Yeo GS, Hung CCC, Rochford J, Keogh J, Gray J, Sivaramakrishnan S, O'Rahilly S \& Farooqi IS 2004 A de novo mutation affecting human TrkB associated with severe obesity and developmental delay. Nature Neuroscience 7 1187-1189. (https://doi.org/10.1038/nn1336)

Young VJ, Brown JK, Saunders PT \& Horne AW 2013 The role of the peritoneum in the pathogenesis of endometriosis. Human Reproduction Update 19 558-569. (https://doi.org/10.1093/humupd/dmt024)

Yu X, Liu L, Cai B, He Y \& Wan X 2008 Suppression of anoikis by the neurotrophic receptor TrkB in human ovarian cancer. Cancer Science $\mathbf{9 9}$ 543-552. (https://doi.org/10.1111/j.1349-7006.2007.00722.x)

Yuan Y, Ye HQ \& Ren QC 2018 Upregulation of the BDNF/TrKB pathway promotes epithelial-mesenchymal transition, as well as the migration and invasion of cervical cancer. International Journal of Oncology 52 461-472. (https://doi.org/10.3892/ijo.2017.4230)

Received 23 March 2020

First decision 21 May 2020

Revised Manuscript received 28 September 2020

Accepted 2 October 2020 